\title{
Segmentation of Fingerprint Images Using Linear Classifier
}

\author{
Xinjian Chen \\ Intelligent Bioinformatics Systems Division, Institute of Automation, The Chinese Academy of Sciences, Beijing 100080, China \\ Email:xjchen@fingerpass.net.cn \\ Jie Tian \\ Intelligent Bioinformatics Systems Division, Institute of Automation, The Chinese Academy of Sciences, Beijing 100080, China \\ Email:tian@doctor.com

\section{Jiangang Cheng} \\ Intelligent Bioinformatics Systems Division, Institute of Automation, The Chinese Academy of Sciences, Beijing 100080, China \\ Email:chengjg@fingerpass.net.cn
}

\author{
Xin Yang \\ Intelligent Bioinformatics Systems Division, Institute of Automation, The Chinese Academy of Sciences, Beijing 100080, China \\ Email:yx@fingerpass.net.cn
}

Received 28 October 2002; Revised 11 September 2003

\begin{abstract}
An algorithm for the segmentation of fingerprints and a criterion for evaluating the block feature are presented. The segmentation uses three block features: the block clusters degree, the block mean information, and the block variance. An optimal linear classifier has been trained for the classification per block and the criteria of minimal number of misclassified samples are used. Morphology has been applied as postprocessing to reduce the number of classification errors. The algorithm is tested on FVC2002 database, only $2.45 \%$ of the blocks are misclassified, while the postprocessing further reduces this ratio. Experiments have shown that the proposed segmentation method performs very well in rejecting false fingerprint features from the noisy background.
\end{abstract}

Keywords and phrases: fingerprint image segmentation, block features, linear classification, image processing.

\section{INTRODUCTION}

The segmentation of fingerprint images is an important step in an automatic fingerprint recognition system. A captured fingerprint image usually consists of two components which are called the foreground and the background. The foreground is the component that originated from the contact of a fingertip with the sensor [1]. The noisy area at the borders of the image is called the background. The aim of segmentation of fingerprint images is to separate the fingerprint foreground area from the background area. Most featureextraction algorithms extract a lot of false features when applied to the noisy background area. So accurate segmentation is especially important for the reliable extraction of features like minutiae and singular points. And after segmentation, the images needed to be enhanced are smaller, so the time needed to enhance is less.

Several approaches to fingerprint image segmentation are known from literature. In [1], Bazen and Gerez proposed a segmentation algorithm based on pixels features, using the criterion of Rosenblatt's perceptron to classify the pixels. The disadvantage of this algorithm is its low speed as it is based on pixels features and moderate performance. The error rate of this algorithm is $6.8 \%$. In [2], the fingerprint is partitioned in blocks of $16 \times 16$ pixels. Then, each block is classified according to the distribution of the gradients in that block. In [3], the previous method is extended by excluding blocks in which gray-scale variance is lower than some threshold. The shortcoming of the above two methods is its moderate segmentation performance. In [4], an adaptive algorithm for uneven background removing at image segmentation base on morphological transformation is presented, but the authors did not give out the detailed experimental results and performance analysis.

In this paper, an algorithm for the segmentation of fingerprints is presented. The algorithm is based on block features so the speed is faster than [1]. The segmentation uses 


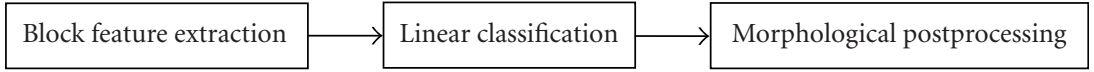

FIGURE 1: Framework of the segmentation algorithm.

three block features, being the block clusters degree, the block mean information, and the block variance. An optimal linear classifier has been trained for the classification per block and the criteria of minimal number of misclassified samples are used. The proposed algorithm has excellent segmentation performance, only $2.45 \%$ of the blocks are misclassified on FVC2002 database (DB), while the postprocessing further reduces this ratio.

This paper is organized as follows. First, Section 2 discusses the block features extraction and linear classification, then Section 3 presents our detailed experimental results; finally, we conclude in Section 4.

\section{BLOCK FEATURES EXTRACTION AND LINEAR CLASSIFICATION}

Steps of our fingerprint segmentation algorithm are shown in Figure 1. The fingerprint is partitioned into blocks of $w \times w$ pixels ( $w=12$ in our algorithm). We select three features that contain useful information for segmentation. These three features are the block clusters degree, the block mean information, and the block variance. An optimal linear classification is used for our segmentation algorithm. Morphological postprocessing is applied to reduce classification errors.

\subsection{Feature extraction}

The aim of feature extraction is to acquire a group of most optimal features for classification. Here we give a criterion for evaluating a feature which is the classification error rate of the feature. The classification error rate Err is computed as follows:

$$
\operatorname{Err}=\frac{N_{\text {err }}}{N_{\text {total }}}=p\left(\omega_{0} \mid \omega_{1}\right)+p\left(\omega_{1} \mid \omega_{0}\right),
$$

where $\omega_{0}$ represent background class, while $\omega_{1}$ represent foreground class.

We select three block features: the block clusters degree, the block mean information, and the block variance. In order to evaluate these features, we randomly select fingerprints as samples in FVC2002 [5] DB3, and these fingerprints had been segmented manually. On the other hand, in order to verify whether these block features can be generalized to segment the fingerprints captured from other sensors, we also select samples from FVC2002 DB1, DB2, and DB4. According to the quality of fingerprints, we select 30 fingerprints in DB3 as samples because of its lower quality, 5 fingerprints in DB1 as samples because of its higher quality, and 10 fingerprints in DB2 and DB4 as samples because of their moderate quality. All of these samples had been segmented manually. In FVC2002, three different scanners and the synthetic fingerprint generator (SFinGe) were used to collect fingerprints

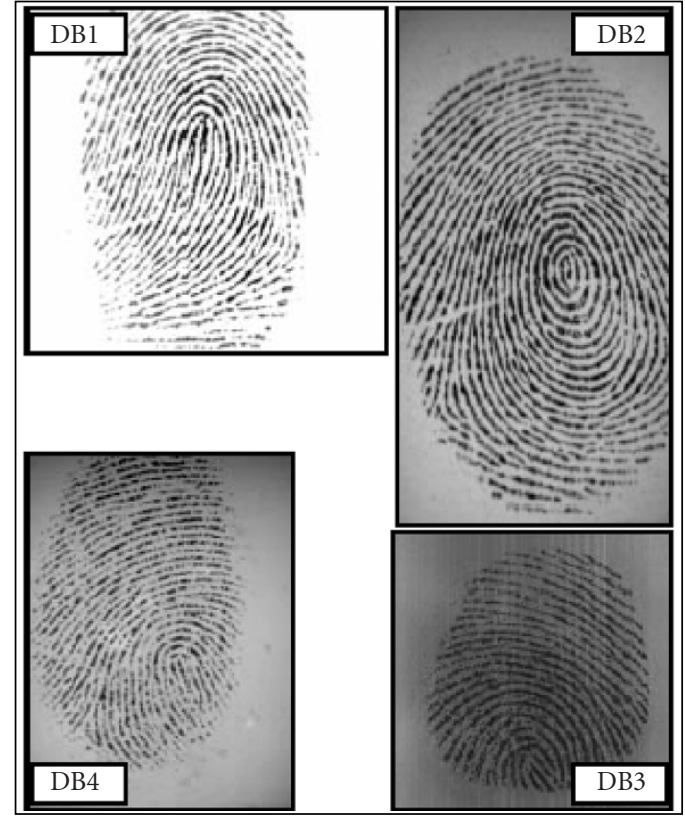

FIGURE 2: One fingerprint image from each database.

(see Table 1). Figure 2 shows an image for each database at the same scale factor. Two examples of fingerprints segmented manually of DB3 are shown in Figure 3.

\subsubsection{The block clusters degree CluD}

The block clusters degree CluD measures how well the ridge pixels are clustering. It is mainly used for this case as in Figure 4.

Using $I$ as the intensity of image, the block clusters degree is defined as follows:

$$
\operatorname{CluD}=\sum_{i, j \in \text { Block }} \operatorname{sign}\left(I_{i j}, \operatorname{Im} g_{\text {mean }}\right) \cdot \operatorname{sign}\left(D_{i j} \text {, Thre } \text { CluD }\right),
$$

where

$$
\begin{gathered}
D_{i j}=\sum_{m=i-2}^{i+2} \sum_{n=j-2}^{j+2} \operatorname{sign}\left(I_{m n}, \operatorname{Im} g_{\text {mean }}\right), \\
\operatorname{sign}(\alpha, \beta)= \begin{cases}1 & \text { if }(\alpha<\beta), \\
0 & \text { otherwise }\end{cases}
\end{gathered}
$$

Im $g_{\text {mean }}$ is the intensity mean of the whole image. The meaning of $D_{i j}$ can be seen in Table 2. Thre ${ }_{\text {CluD }}$ is an empirical parameter, Thre $_{\mathrm{CluD}}=15$ in our algorithm. 
TABLE 1: Scanners/technologies used for the collection of FVC2002 databases.

\begin{tabular}{lllr}
\hline DB & Technology & Scanner & Image size resolution \\
\hline DB1 & Optical & Identix TouchView II & $388 \times 374,500 \mathrm{dpi}$ \\
DB2 & Optical & Biometrika FX2000 & $296 \times 560,569 \mathrm{dpi}$ \\
DB3 & Capacitive & Precise Biometrics 100SC & $300 \times 300,500 \mathrm{dpi}$ \\
DB4 & Synthetic & SFinGe v2.51 & $288 \times 384,500 \mathrm{dpi}$ \\
\hline
\end{tabular}

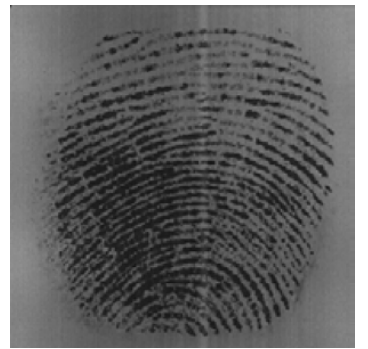

(a) Original fingerprint.

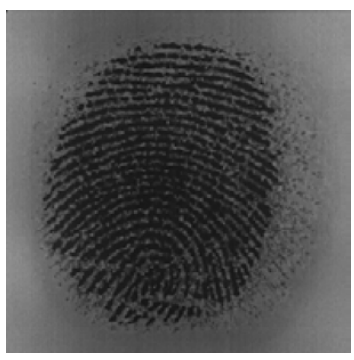

(c) Original fingerprint.

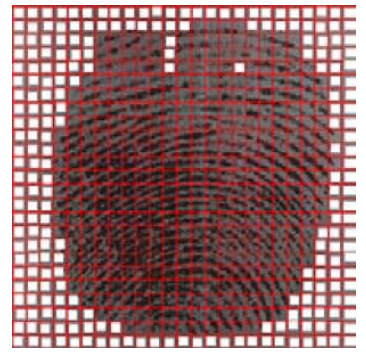

(b) Fingerprint segmented manually.

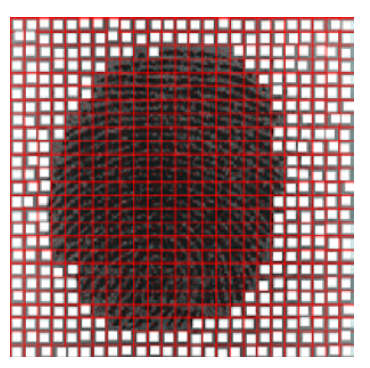

(d) Fingerprint segmented manually.
FIGURE 3: Two examples of fingerprints and segmented fingerprints of DB3.

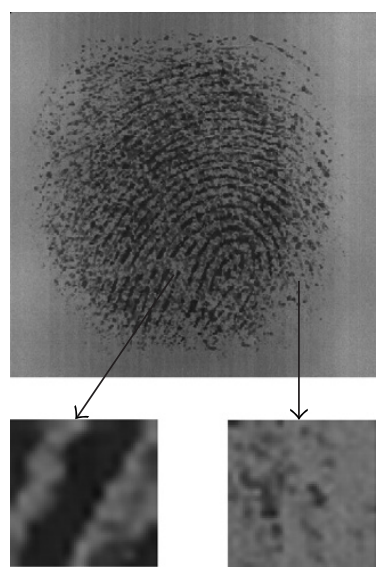

(a)

(b)

FIgure 4: The illustration of block clusters degree: (a) CluD is bigger and (b) CluD is smaller.

As we select 30 samples in DB3, the size of DB3 fingerprint images is $300 \times 300$, hence the total number of the sam-
TABLE 2: 25 pixels centered $p_{i j}$ for computing $D_{i j}$.

\begin{tabular}{|c|c|c|c|c|}
\hline$P_{i-2 j-2}$ & $P_{i-2 j-1}$ & $P_{i-2 j}$ & $P_{i-2 j+1}$ & $P_{i-2 j+2}$ \\
\hline$P_{i-1 j-2}$ & $P_{i-1 j-1}$ & $P_{i-1 j}$ & $P_{i+1 j+1}$ & $P_{i-1 j+2}$ \\
\hline$P_{i j-2}$ & $P_{i j-1}$ & $P_{i j}$ & $P_{i j+1}$ & $P_{i j+2}$ \\
\hline$P_{i+1 j-2}$ & $P_{i+1 j-1}$ & $P_{i+1 j}$ & $P_{i+1 j+1}$ & $P_{i+1 j+2}$ \\
\hline$P_{i+2 j-2}$ & $P_{i+2 j-1}$ & $P_{i+2 j}$ & $P_{i+2 j+1}$ & $P_{i+2 j+2}$ \\
\hline
\end{tabular}

ples' blocks is $(300 / 12) \times(300 / 12) \times 30=625 \times 30=18750$. From Figure 5, we can find that the feature of block clusters degree has excellent classification performance for DB3. When threshold $=2$, we can get the minimal error rate Err of this feature as Err $=1218 / 18750=0.06496$.

This block feature is also used for segmenting the fingerprint images captured from other sensors.

(1) As we select 5 samples in DB1, the size of DB1 fingerprint images is $388 \times 374$, hence the total number of the samples' blocks is $(388 / 12) \times(374 / 12) \times 5=1056 \times 5=5280$. Figure 6 show the feature of block clusters degree of DB1 samples. When threshold $=1$, we can get the minimal error rate Err of this feature as Err $=577 / 5280=0.10928$.

(2) As we select 10 samples in DB2, the size of DB2 fingerprint images is $296 \times 560$, hence the total number of the samples' blocks is $(296 / 12) \times(560 / 12) \times 10=1175 \times 10=11750$. Figure 7 show the feature of block clusters degree of DB2 samples. When threshold $=1$, we can get the minimal error rate Err of this feature as Err $=568 / 11750=0.04834$.

(3) As we select 10 samples in DB4, the size of DB4 fingerprint images is $288 \times 384$, hence the total number of the samples' blocks is $(288 / 12) \times(384 / 12) \times 10=768 \times 10=7680$. Figure 8 show the feature of block clusters degree of DB4 samples. When threshold $=1$, we can get the minimal error rate Err of this feature as Err $=781 / 7680=0.10169$.

\subsubsection{The block mean information Meanl}

For most fingerprint sensors, the ridge-valley structures can be approximated by black and white lines, while the background, where the finger does not touch the sensor, is rather white. This means that the mean gray value in the foreground is in general lower, that is, darker gray, than it is in the background. But in fact there are always some fingerprints that are too wet or too dry. Examples are shown in Figure 9. So we cannot only use the block mean, we should take into account the mean intensity of the whole image. We use the difference of local block mean and global image mean as the second feature for fingerprints segmentation. 

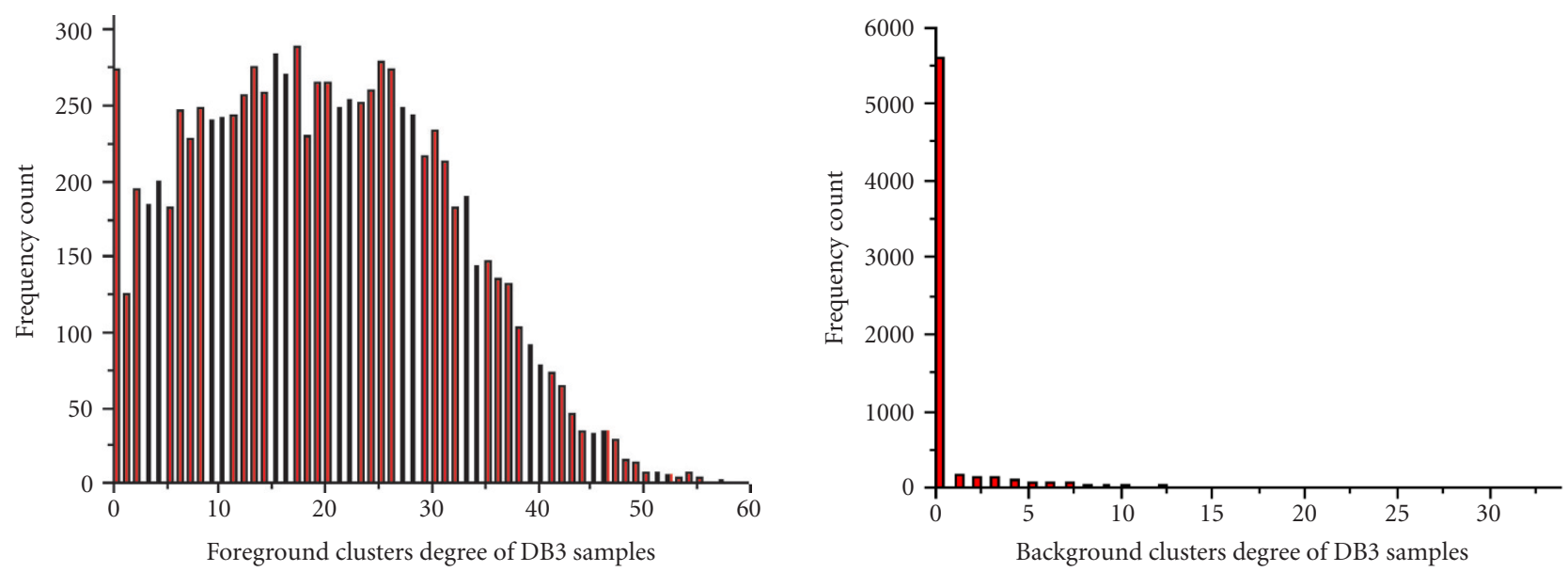

Figure 5: The block clusters degree CluD of the samples. The horizontal coordinate represents the value of the block clusters degree, while the vertical coordinate represents the frequency count of the value.
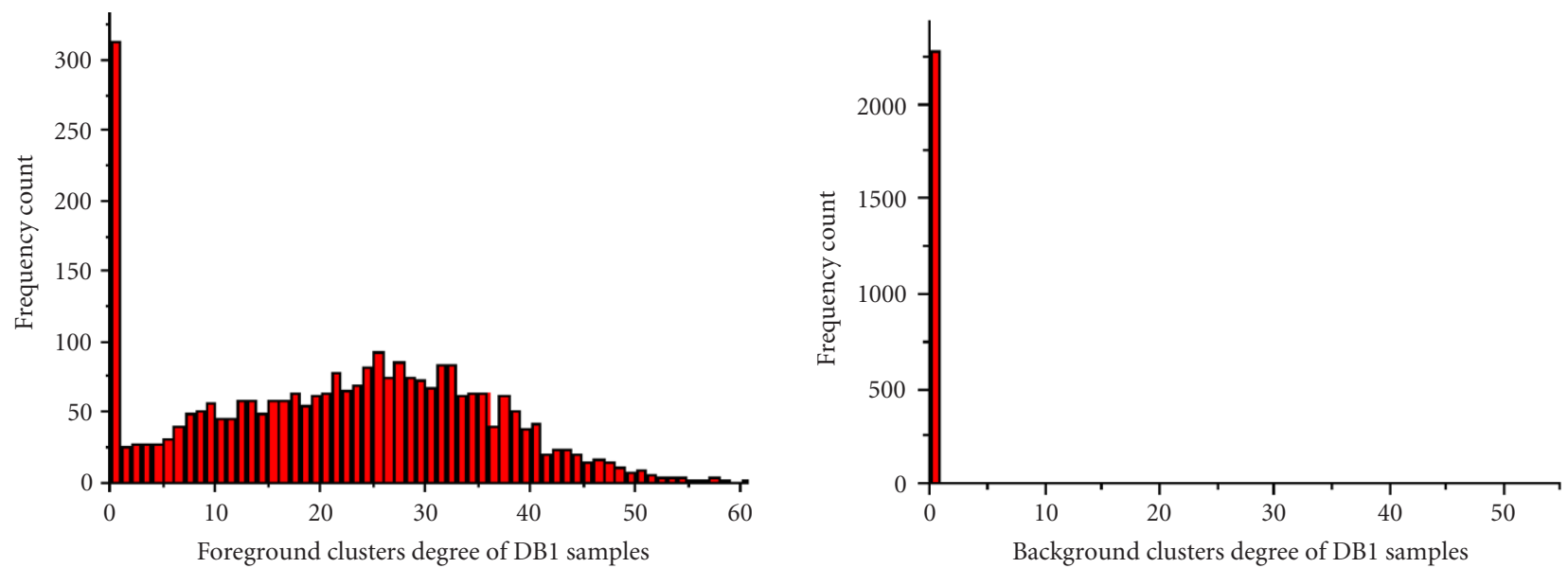

Figure 6: The block clusters degree CluD of the samples. The horizontal coordinate represents the value of the block clusters degree while the vertical coordinate represents the frequency count of the value.
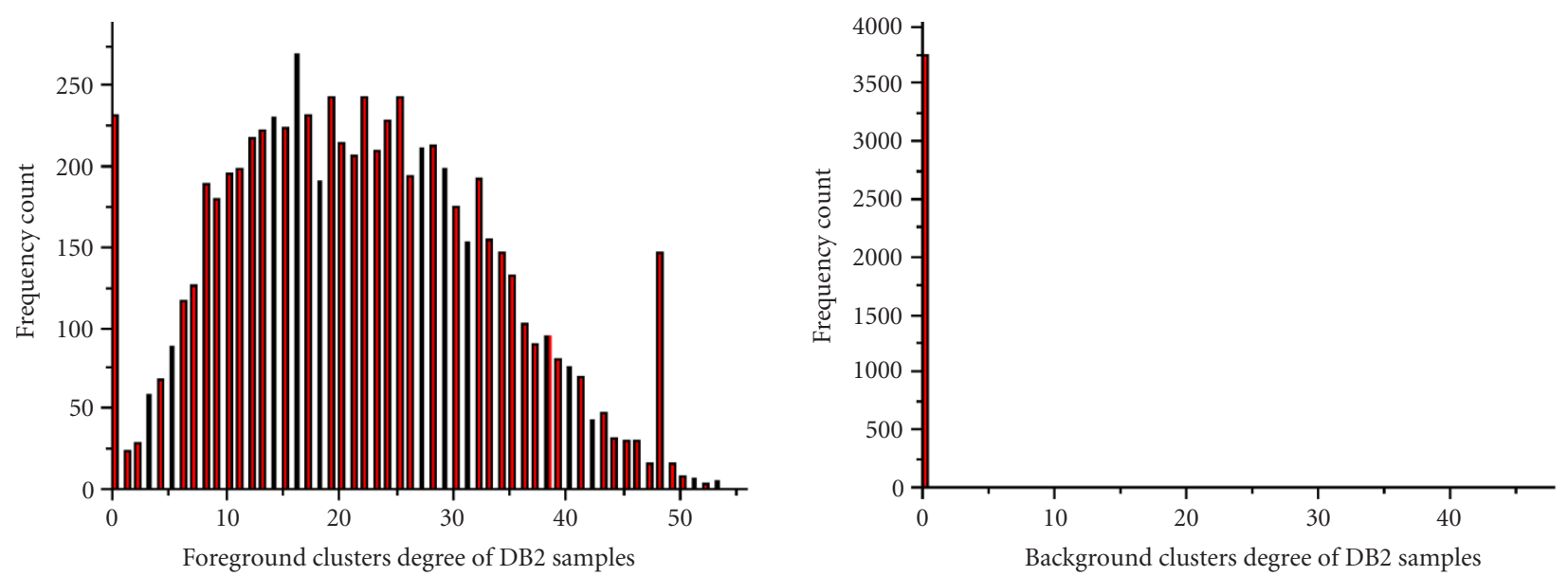

Figure 7: The block clusters degree CluD of the samples. The horizontal coordinate represents the value of the block clusters degree while the vertical coordinate represents the frequency count of the value. 

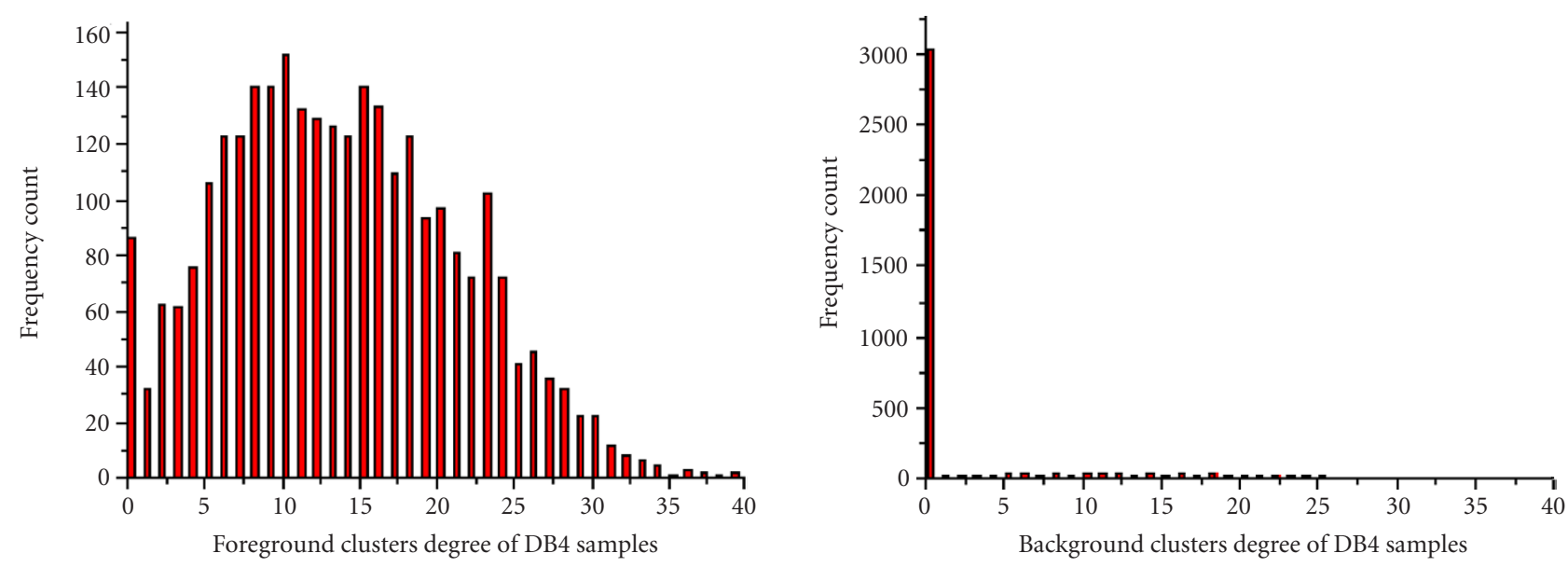

FIGURE 8: The block clusters degree CluD of the samples. The horizontal coordinate represents the value of the block clusters degree while the vertical coordinate represents the frequency count of the value.

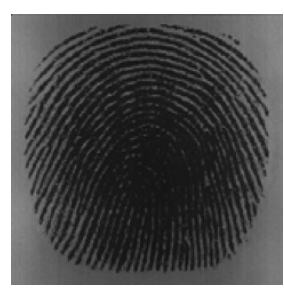

(a)

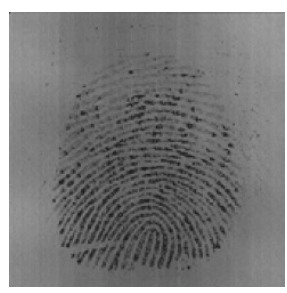

(b)
FIgURE 9: Examples of fingerprint: (a) too wet and (b) too dry.

The mean information MeanI for each block is given by

$$
\text { MeanI }=\left(\frac{1}{w \cdot w} \sum_{\text {Block }} I\right)-\operatorname{Im} g_{\text {mean }}
$$

From Figure 10, we also can find that the feature of block mean information have good classification performance for DB3. When threshold $=14.5$, we can get the minimal error rate Err of this feature as $\operatorname{Err}=2294 / 18750=0.12230$.

On the other hand, we also use block mean to segment the fingerprints. In Figure 11, the feature of block mean of DB3 samples are shown. When threshold $=101$, we can get the minimal error rate Err of this feature as Err = $2662 / 18750=0.14197$. From Figures 10 and 11 , we can find that block mean information MeanI has better classifier performance than block mean.

This block feature is also used for segmenting the fingerprint images captured from other sensors.

(1) Figure 12 shows the feature of block mean information of DB1 samples. When threshold $=16.5$, we can get the minimal error rate Err of this feature as Err $=858 / 5280=$ 0.16250 .

(2) Figure 13 shows the feature of block mean information of DB2 samples. When threshold $=15.5$, we can get the minimal error rate Err of this feature as Err $=1826 / 11750=$ 0.15540 .
(3) Figure 14 shows the feature of block mean information of DB4 samples. When threshold $=18.5$, we can get the minimal error rate Err of this feature as Err $=1035 / 7680=$ 0.13476 .

\subsubsection{The block variance Var}

The block variance Var is the third feature that is used. In general, the variance of the ridge-valley structures in the foreground is higher than the variance of the noise in the background. The block variance Var for each block is given by

$$
\text { Var }=\frac{1}{w \cdot w} \sum_{\text {Block }}(I-\text { mean })^{2} .
$$

From Figure 15, we can also find that the feature of block variance have excellent classification performance for DB3. When threshold $=323$, we can get the minimal error rate Err of this feature as Err $=1396 / 18750=0.07445$.

This block feature is also used for segmenting the fingerprint images from other kinds of sensors.

(1) Figure 16 shows the feature of block variance of DB1 samples. When threshold $=486$, we can get the minimal error rate Err of this feature: Err $=536 / 5280=0.10151$.

(2) Figure 17 shows the feature of block variance of DB2 samples. When threshold $=165$, we can get the minimal error rate Err of this feature: Err $=1159 / 11750=0.09863$.

(3) Figure 18 shows the feature of block variance of DB4 samples. When threshold $=190$, we can get the minimal error rate Err of this feature as Err $=608 / 7680=0.07916$.

\subsubsection{Summary of block features}

Usually, fingerprints captured from different kinds of sensors have different characters. From Table 3, we can find that CluD has better classification performance for DB2, but Var has better classification performance for DB4; and CluD and Var play an equivalently important role in segmention for DB1 and DB3. 

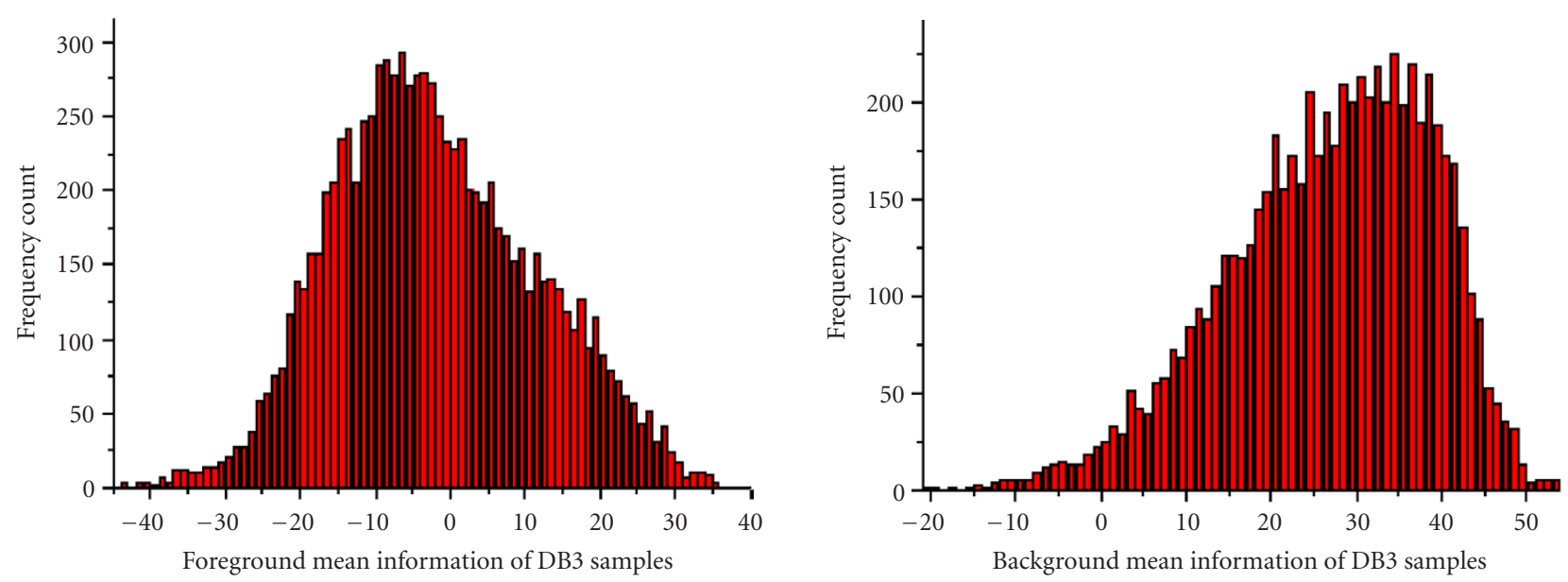

FIGURE 10: The block mean information MeanI of the samples. The horizontal coordinate represents the value of the block mean information while the vertical coordinate represents the frequency count of the value.
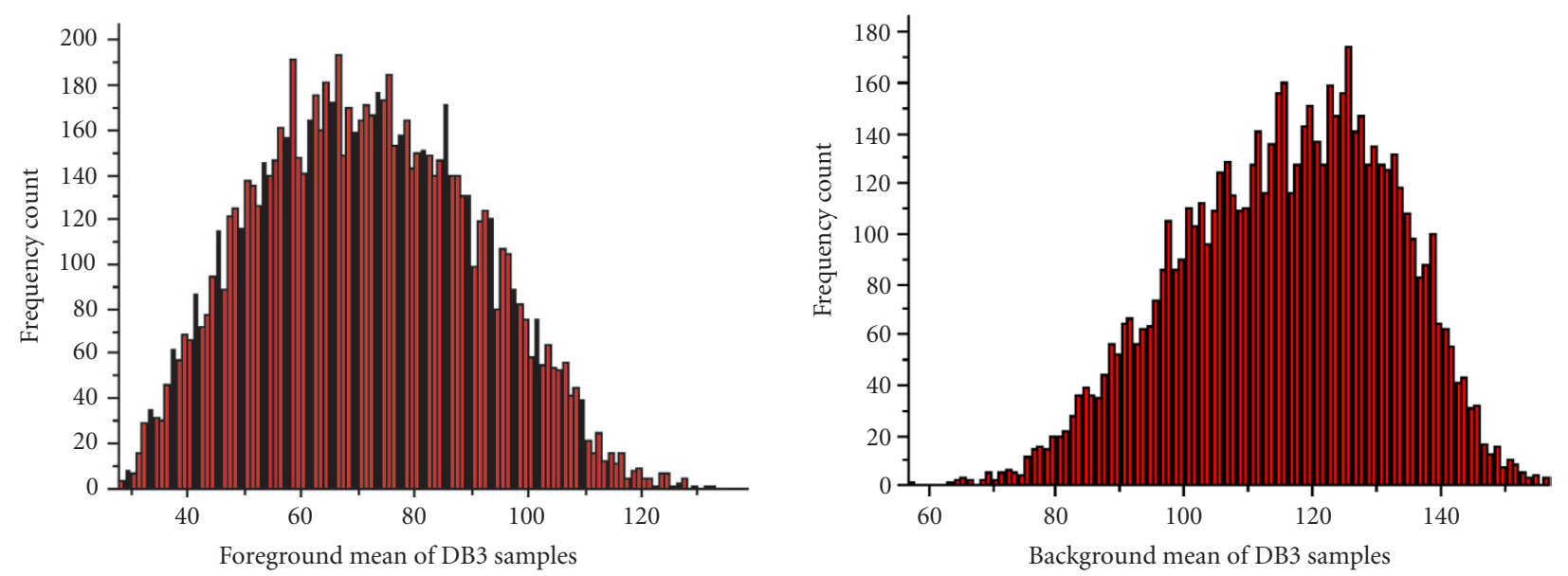

Figure 11: The block mean of the samples. The horizontal coordinate represents the value of the block mean while the vertical coordinate represents the frequency count of the value.
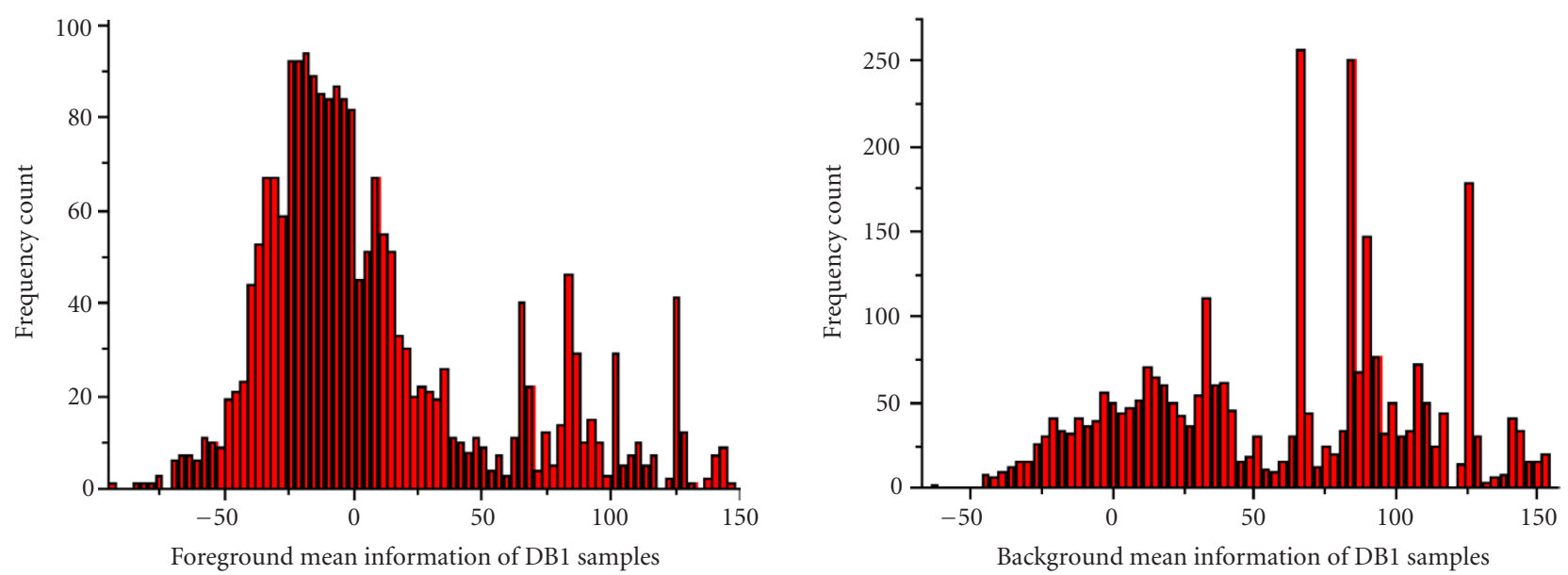

FIgURE 12: The block mean information MeanI of the samples. The horizontal coordinate represents the value of the block mean information while the vertical coordinate represents the frequency count of the value. 

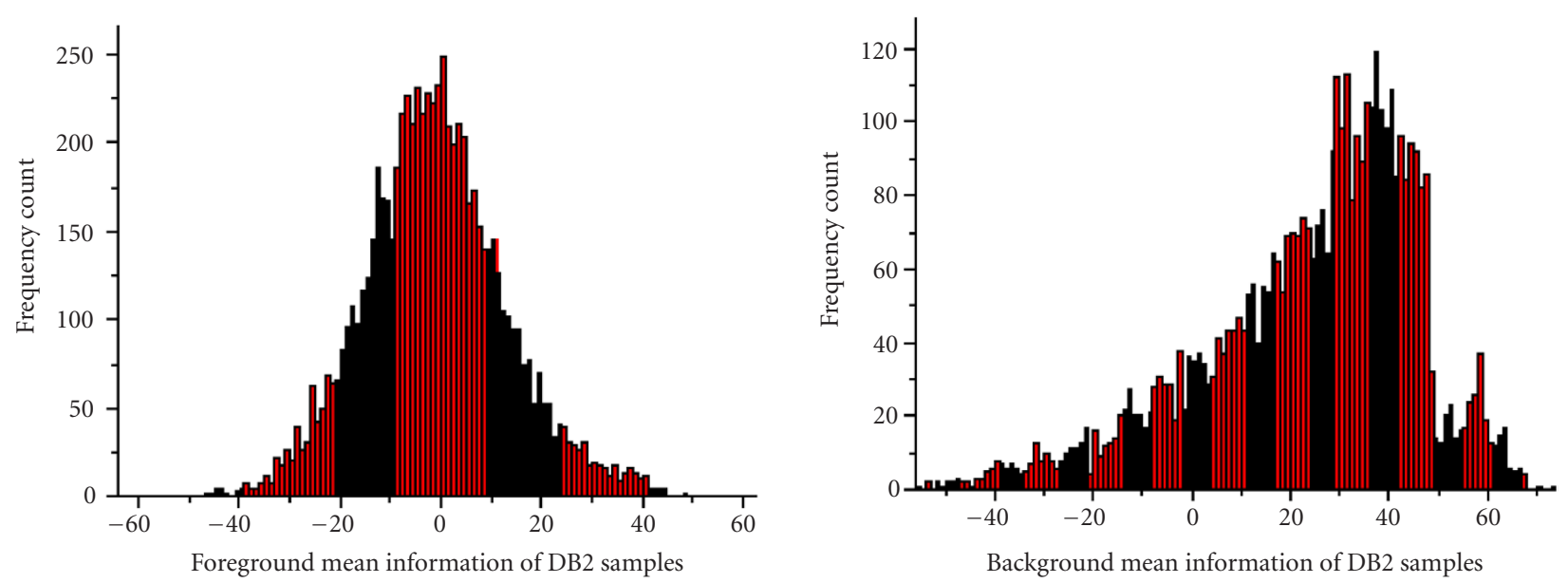

FIGURE 13: The block mean information MeanI of the samples. The horizontal coordinate represents the value of the block mean information while the vertical coordinate represents the frequency count of the value.
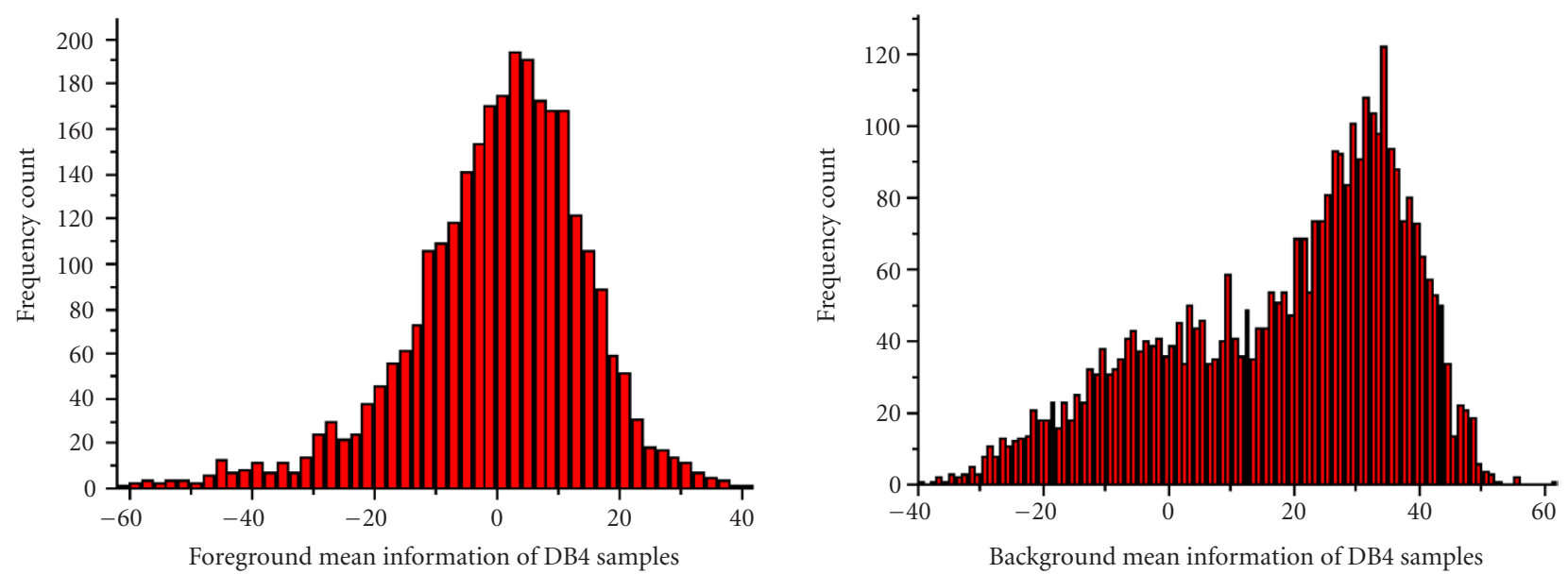

FIGURE 14: The block mean information MeanI of the samples. The horizontal coordinate represents the value of the block mean information while the vertical coordinate represents the frequency count of the value.
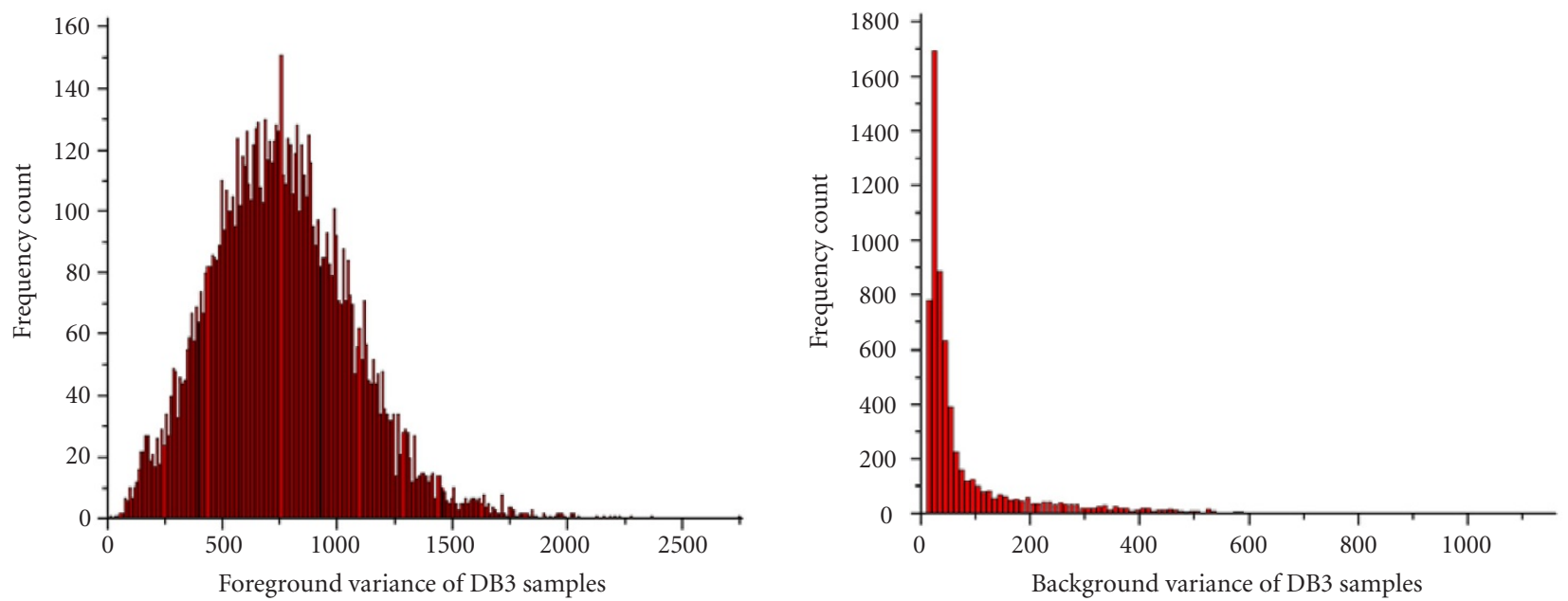

FIGURE 15: The block variance Var of the samples. The horizontal coordinate represents the value of the block variance while the vertical coordinate represents the frequency count of the value. 

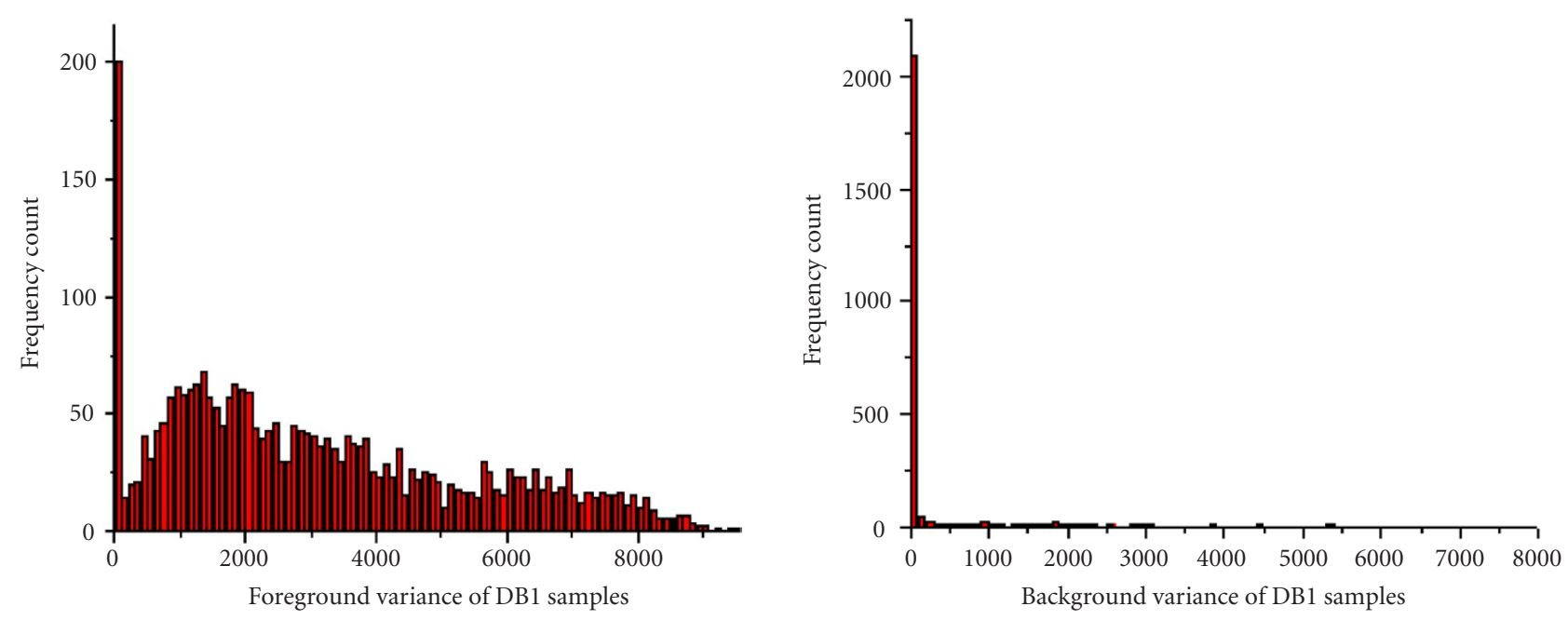

FIGURE 16: The block variance Var of the samples. The horizontal coordinate represents the value of the block variance while the vertical coordinate represents the frequency count of the value.
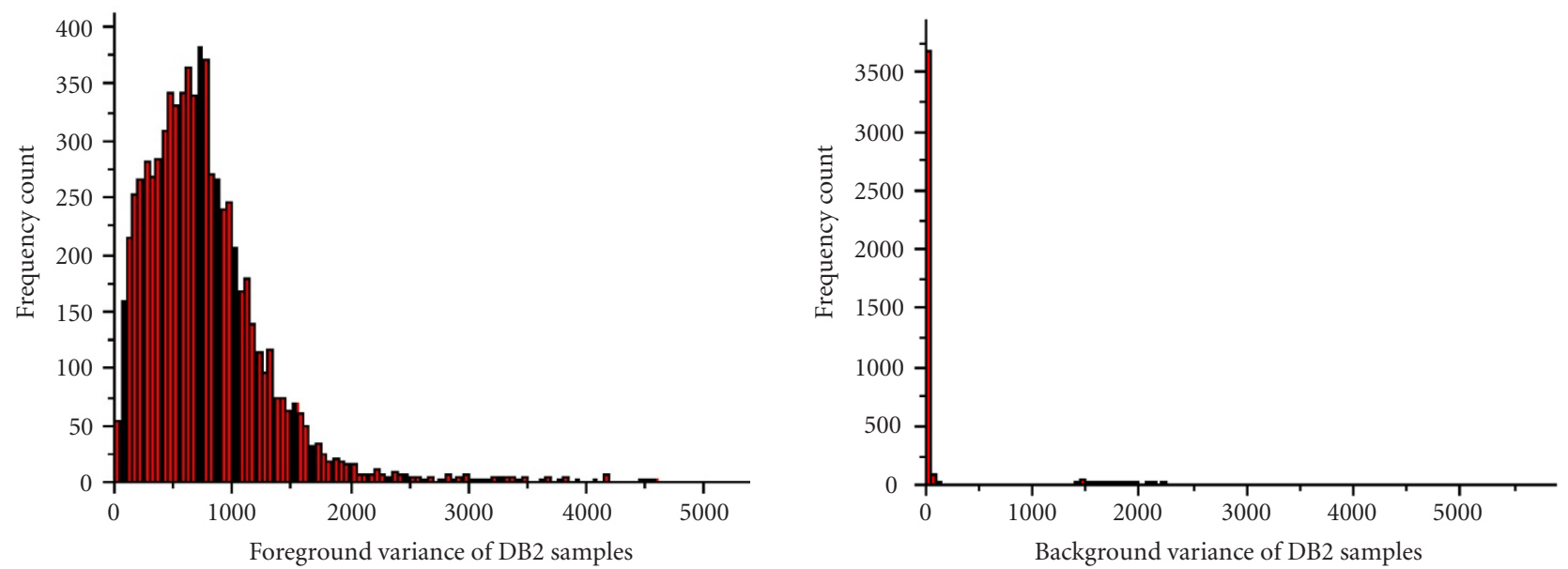

FIGURE 17: The block variance Var of the samples. The horizontal coordinate represents the value of the block variance while the vertical coordinate represents the frequency count of the value.
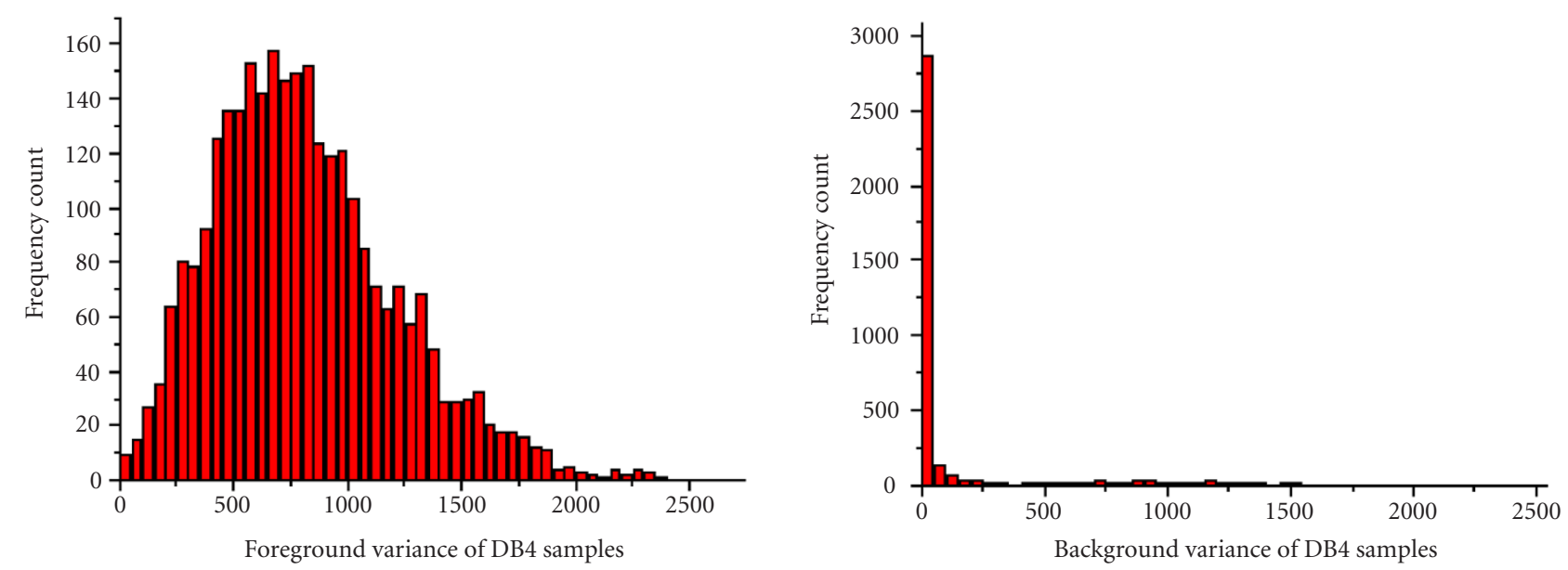

FIGURE 18: The block variance Var of the samples. The horizontal coordinate represents the value of the block variance while the vertical coordinate represents the frequency count of the value. 
TABLE 3: Summary of block features Err for each DB.

\begin{tabular}{lccc}
\hline DB & CluD & MeanI & Var \\
\hline DB1 & 0.10928 & 0.16250 & 0.10151 \\
DB2 & 0.04834 & 0.15540 & 0.09863 \\
DB3 & 0.06496 & 0.12230 & 0.07445 \\
DB4 & 0.10169 & 0.13476 & 0.07916 \\
\hline
\end{tabular}

\subsection{Linear classification design}

In this paper, we will follow a supervised approach since the block features of samples in both areas are available. Using this method, a classification algorithm can be constructed that minimizes the probability of misclassifying. Many different classification algorithms exist that can be applied to this problem. One can for instance think of $K$-nearest neighbor, neural networks, and so forth to find the optimal decision boundaries [6]. However, it is very important to use a classification algorithm that has the lowest computational complexity possible. We have therefore chosen to use a linear classifier which tests a linear combination of the features given by

$$
v=w^{T} x=w_{0} \mathrm{CluD}+w_{1} \text { MeanI }+w_{2} \operatorname{Var}+w_{3},
$$

where $v$ is the value to be tested, $w=\left[\begin{array}{llll}w_{0} & w_{1} & w_{2} & w_{3}\end{array}\right]^{T}$ is the weight vector, and $x=[\mathrm{CluD} \text { MeanI } \operatorname{Var} 1]^{T}$ is the feature vector. Then, using class $\omega_{1}$ for the foreground, class $\omega_{0}$ for the background, and $\hat{\omega}$ for the assigned class, the following decision function is applied:

$$
\hat{\omega}= \begin{cases}\omega_{1} & \text { if } w^{T} x>0 \\ \omega_{0} & \text { otherwise }\end{cases}
$$

If the samples are two linearly separable classes, we know that there exists a vector $w$, satisfying

$$
\begin{array}{ll}
w^{T} x>0 & \forall x \in \omega_{1}, \\
w^{T} x<0 & \forall x \in \omega_{0} .
\end{array}
$$

So we let

$$
x_{n}^{\prime}= \begin{cases}x_{i} & \forall x_{i} \in \omega_{1}, \\ -x_{i} & \forall x_{i} \in \omega_{0},\end{cases}
$$

then our task is to find a weight vector $w$, where

$$
w^{T} x_{n}^{\prime}>0, \quad n=1,2, \ldots, N ;
$$

here $N$ is the number of samples.

In [1], the criterion of Rosenblatt's perceptron is used to classify the pixels. But the criterion of Rosenblatt's perceptron is only suited for linearly separable classes, and generally, samples are not linearly separable, so the classification performance of [1] is moderate. In our algorithm, we use the criteria of minimal number of misclassified samples [7] to classify the blocks.
Using the form of matrix, (10) can be written as follows:

$$
X w>0,
$$

where

$$
X=\left[\begin{array}{c}
x_{1}^{T} \\
x_{2}^{T} \\
\vdots \\
x_{N}^{T}
\end{array}\right]=\left[\begin{array}{cccc}
x_{11} & x_{12} & \cdots & x_{14} \\
x_{21} & x_{22} & \cdots & x_{24} \\
\vdots & \vdots & \vdots & \vdots \\
x_{N 1} & x_{N 2} & \cdots & x_{N 4}
\end{array}\right]
$$

In order to make the solution more credible, let

$$
X w \geq b>0 .
$$

In general, we let

$$
b=\left[\begin{array}{c}
1 \\
1 \\
\vdots \\
1
\end{array}\right]_{N \times 1}
$$

Then the criteria function can be defined as follows:

$$
J(w)=\|(X w-b)-|x w-b|\|^{2} .
$$

If $X w \geq b$, then $J(w)=0$, otherwise $J(w)>0$. So the more the number of samples unsatisfied are, the larger the value of $J(w)$ is. Then our aim is to find a vector $w$ to make the value of $J(w)$ minimal. We use the conjugate gradient algorithm [8]; for the detailed steps of algorithm see [8].

\subsection{Postprocessing}

Unlike other images, fingerprint image has its own characteristics [9]. It is valuable to introduce human knowledge into the processing and postprocessing of the fingerprint images. More compact clusters can be obtained by a number of different postprocessing methods. It is possible to use either boundary-based methods like curve fitting and active contour models, or region-based methods like region growing and morphology [10]. We have chosen to apply morphology to the classification estimate. It reduces the number of false classifications. First, small clusters that are incorrectly assigned to the foreground are removed by means of an open operation [4]. Next, small clusters that are incorrectly assigned to the background are removed by a close operation. After the morphological processing, we connect the edges and corners using the lines.

Two examples of the postprocessing are shown in Figure 19. The segmented result is the fingerprint image bounded by blue line.

\section{EXPERIMENTAL RESULTS}

The segmentation algorithm was tested on 4 databases of FVC2002. All the experiments were done in Pentium 4 CPU 


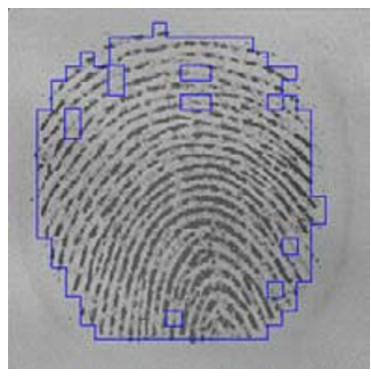

(a) Before postprocessing.

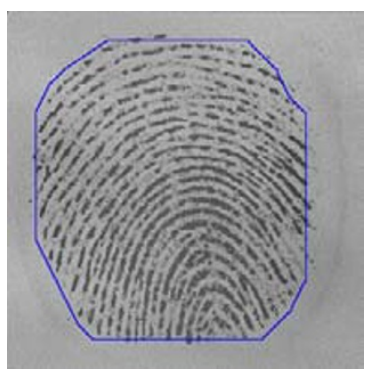

(b) After postprocessing.

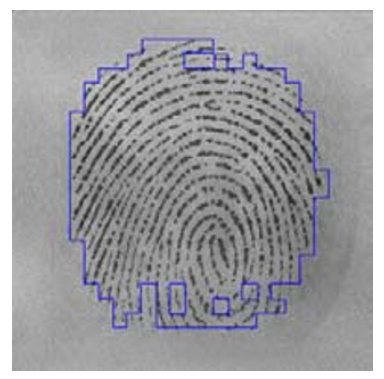

(c) Before postprocessing.

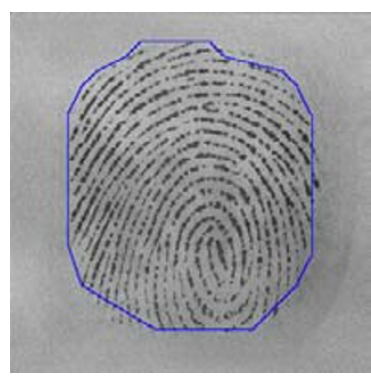

(d) After postprocessing.

Figure 19: Two examples of the postprocessing.

TABle 4: Segmentation time in P4 2.4 GHz PC for each DB (seconds).

\begin{tabular}{l|cccc}
\hline Segmentation time & DB1 & DB2 & DB3 & DB4 \\
\hline $\begin{array}{l}\text { Segmentation time of } \\
\text { our algorithm (s) }\end{array}$ & 0.018 & 0.019 & 0.015 & 0.016 \\
\hline $\begin{array}{l}\text { Segmentation time used in } \\
\text { the algorithm in [1] (s) }\end{array}$ & 0.125 & 0.145 & 0.094 & 0.110 \\
\hline
\end{tabular}

2.4 GHz PC. Table 4 gives the time needed to segment a fingerprint image for each DB of FVC2002. Meanwhile, in order to compare the proposed algorithm with [1], we have done some experiments that used the algorithm in [1]. From Table 4, we can conclude that our algorithm is enormously faster than [1].

\subsection{The result of FVC2002 DB3}

Firstly, the segmentation algorithm has been trained on these 30 fingerprint samples. The weight vector of the trained results is

$$
w^{T}=\left(w_{0}, w_{1}, w_{2}, w_{3}\right)=(1.152,-0.433,0.067,-24.0) .
$$

Then we use this weight vector for classification by expression (7), the computed results is shown in Figure 20. We can find that our classifier have excellent classification performance.

In Figure 21, segmentation results are shown for three fingerprints from FVC2002 DB3 using the proposed algorithm. Figure $21 \mathrm{a}$ is from the training data, while Figures $21 \mathrm{~b}$ and $21 \mathrm{c}$ are from the test data. Human inspection shows that our algorithm provides satisfactory results. Meanwhile in Figure 22, we have given out segmentation results of the same three fingerprints using the algorithm in [1]. From Figure 22, we find that the segmentation results of our algorithm are better than the results of [1].

Apart from human inspection, we can quantitatively evaluate the results of a segmentation algorithm. The number of classification errors could be used as a performance measure. This is exactly the measure that was used during training:

$$
\begin{aligned}
p\left(\omega_{0} \mid \omega_{1}\right) & =\frac{\text { num }_{\text {error classification }}}{\text { num }_{\text {total foreground blocks }}} \\
& =\frac{335}{9309}=0.0359, \\
p\left(\omega_{1} \mid \omega_{0}\right) & =\frac{\text { num }_{\text {error classification }}}{\text { num }_{\text {total background blocks }}} \\
& =\frac{328}{9441}=0.0347, \\
\text { Err } & =\frac{\text { num }_{\text {error classification }}}{\text { num }_{\text {total blocks }}}=\frac{663}{18750}=0.0353 .
\end{aligned}
$$

Here Err is the value before morphological postprocessing; after postprocessing, the error rate will become smaller.

\subsection{The result of FVC2002 DB1}

Using the method above, the weight vector of trained results is

$$
w^{T}=\left(w_{0}, w_{1}, w_{2}, w_{3}\right)=(3.723,-0.389,0.071,-12.6) .
$$

The computed results are shown in Figure 23 and segmentation results are shown for three fingerprints from FVC2002 DB1 in Figure 24.

The error rate of DB1 is the following:

$$
\begin{aligned}
p\left(\omega_{0} \mid \omega_{1}\right) & =\frac{39}{2802}=0.0139 \\
p\left(\omega_{1} \mid \omega_{0}\right) & =\frac{56}{2478}=0.0225 \\
\text { Err } & =\frac{95}{5280}=0.0180
\end{aligned}
$$

\subsection{The result of FVC2002 DB2}

The weight vector of trained results is

$$
\begin{aligned}
w^{T} & =\left(w_{0}, w_{1}, w_{2}, W_{3}\right) \\
& =(2.342,-0.793,0.046,-11.9)
\end{aligned}
$$

The computed results are shown in Figure 25 and segmentation results are shown for three fingerprints from FVC2002 DB2 in Figure 26. 


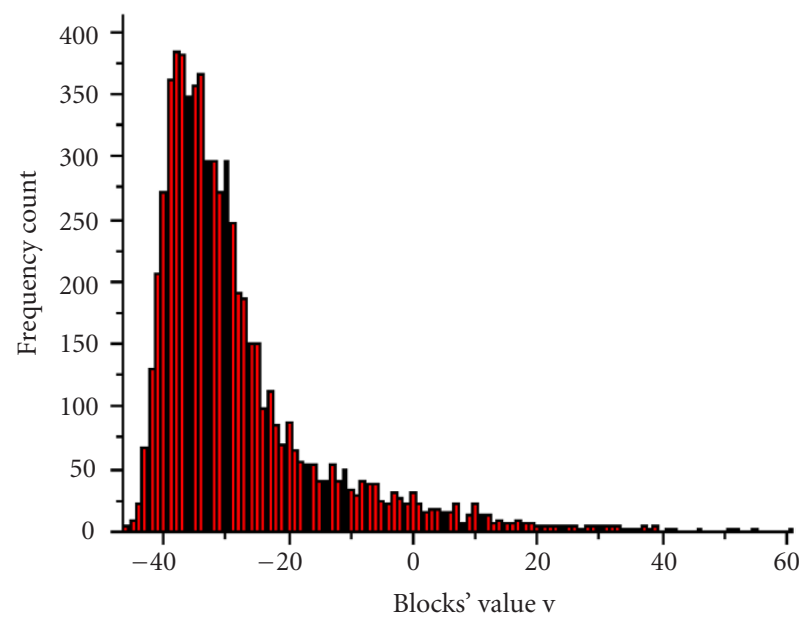

(a)

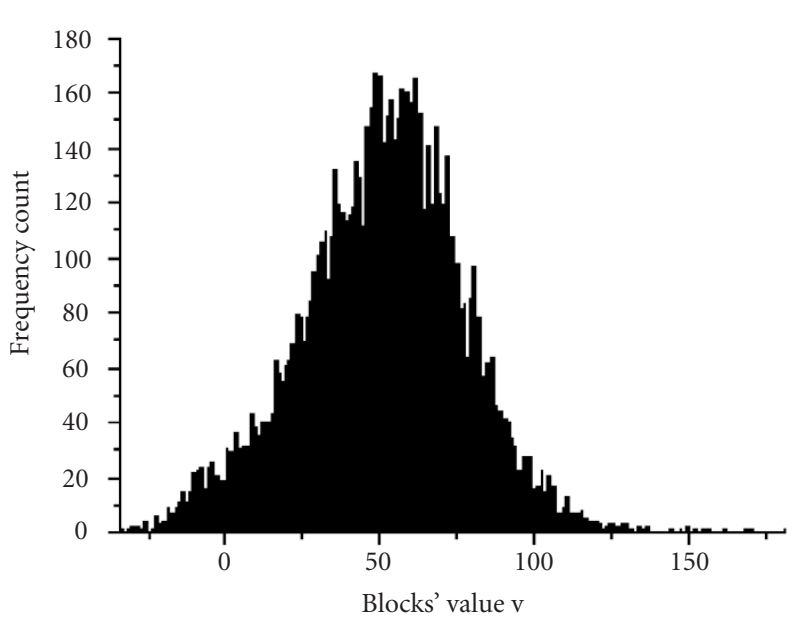

(b)

FIgURE 20: The value of the (a) background and (b) foreground class in the linear classification in FVC2002 DB3.

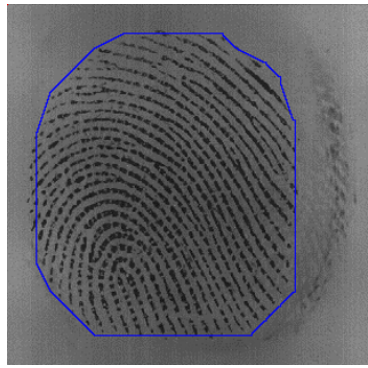

(a)

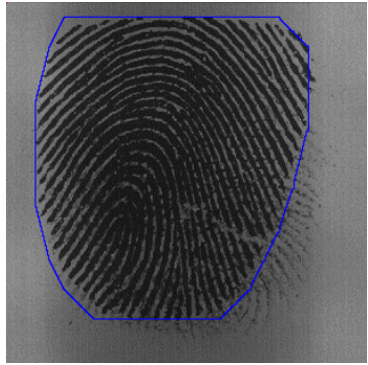

(b)

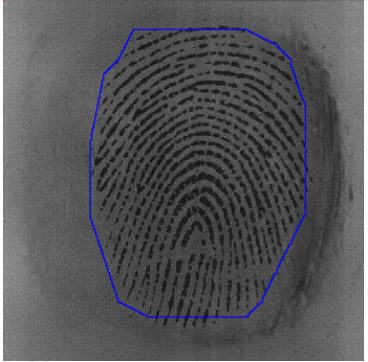

(c)

Figure 21: Segmentation results of three fingerprints from FVC2002 DB3 using our algorithm: (a) is from the training data, (b) and (c) are from the test data.
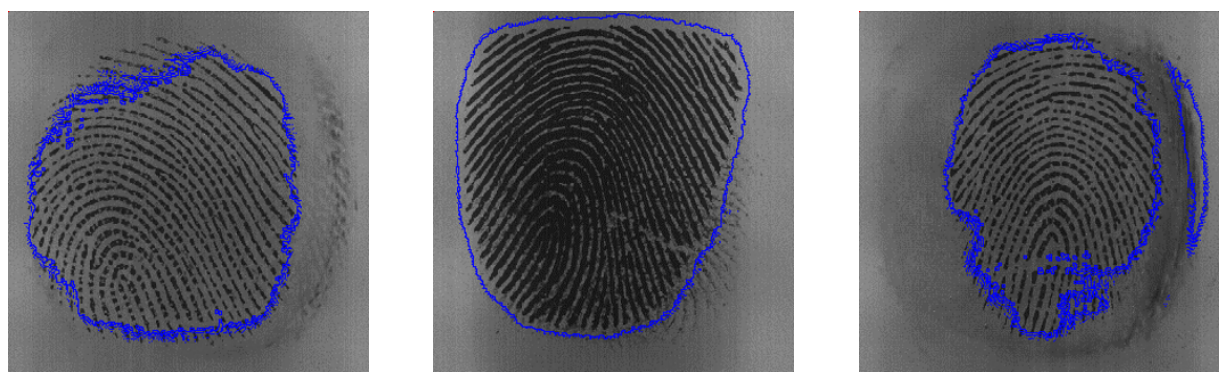

FIGURE 22: Segmentation results of three fingerprints from FVC2002 DB3 using the algorithm in [1].

The error rate of DB2 is the following:

$$
\begin{aligned}
p\left(\omega_{0} \mid \omega_{1}\right) & =\frac{226}{7346}=0.0307, \\
p\left(\omega_{1} \mid \omega_{0}\right) & =\frac{118}{4404}=0.0268, \\
\text { Err } & =\frac{344}{11750}=0.0293 .
\end{aligned}
$$

\subsection{The result of FVC2002 DB4}

The weight vector of trained results is

$$
\begin{aligned}
w^{T} & =\left(w_{0}, w_{1}, w_{2}, w_{3}\right) \\
& =(5.701,-0.263,0.036,-10.5) .
\end{aligned}
$$

The computed results are shown in Figure 27 and segmentation results are shown for three fingerprints from FVC2002 DB4 in Figure 28. 


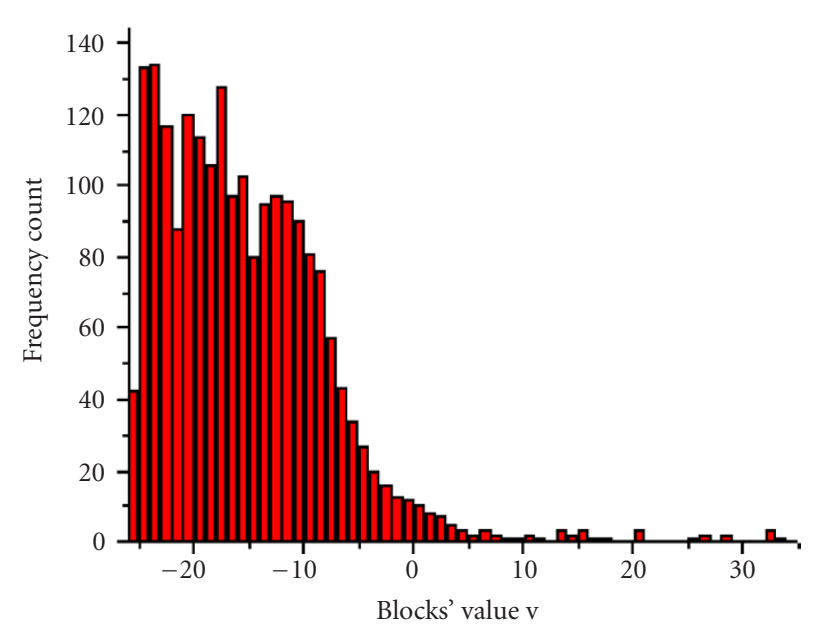

(a)

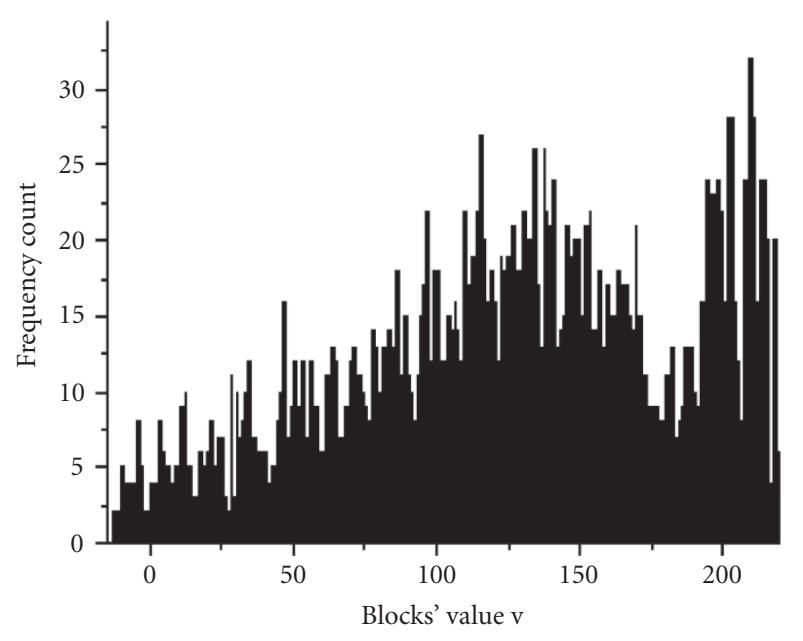

(b)

FIGURE 23: The value of the (a) background and (b) foreground class in linear classification in FVC2002 DB1.

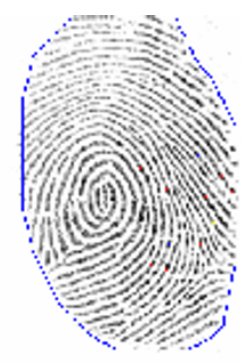

(a)

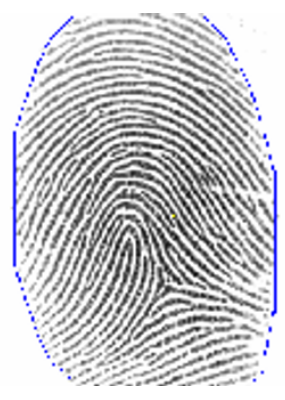

(b)

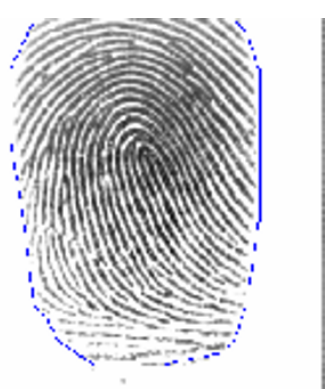

(c)

FIGURE 24: Segmentation results of three fingerprints from FVC2002 DB1: (a) is from the training data, (b) and (c) are from the test data.

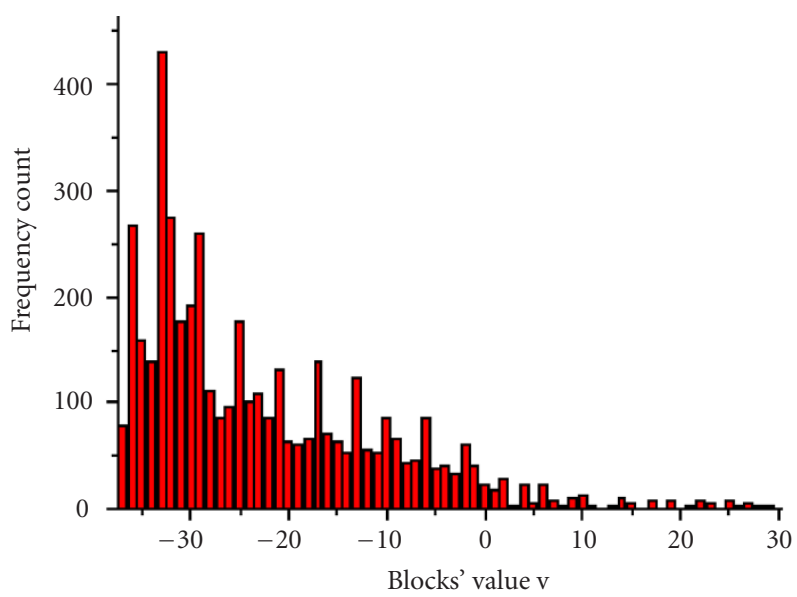

(a)

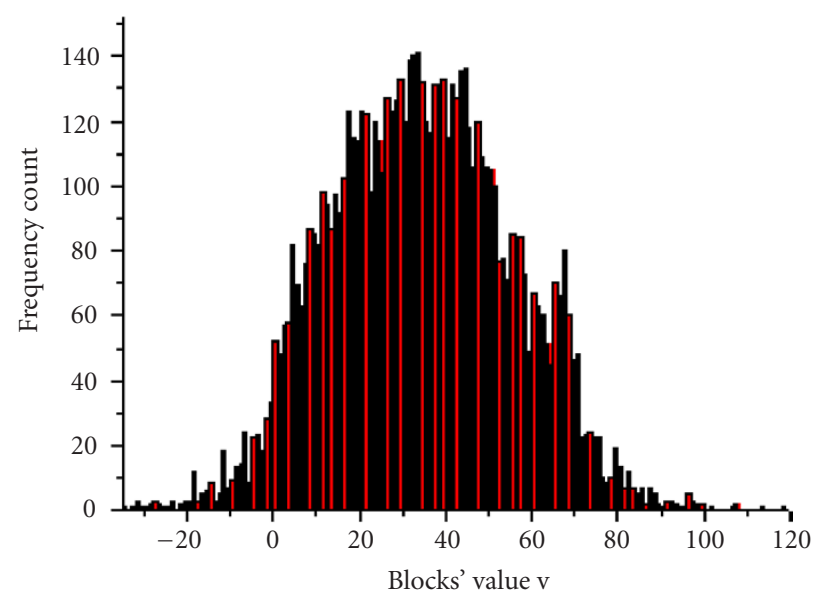

(b)

FIGURE 25: The value of the (a) background and (b) foreground class in linear classification in FVC2002 DB2. 


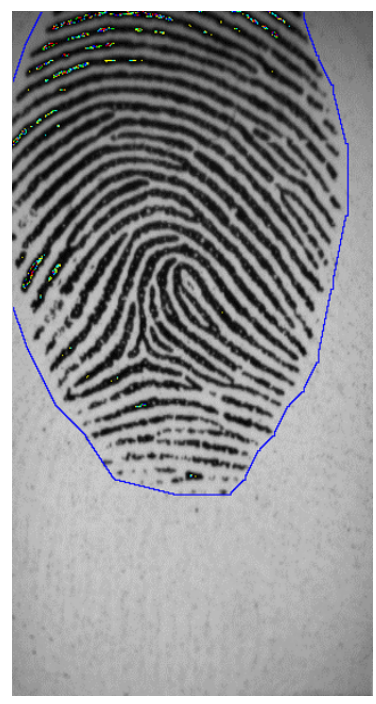

(a)

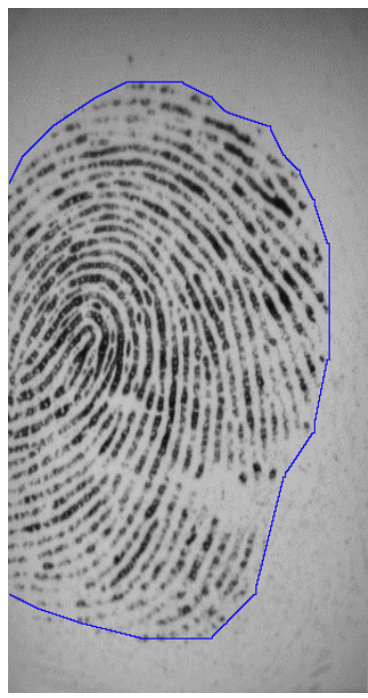

(b)

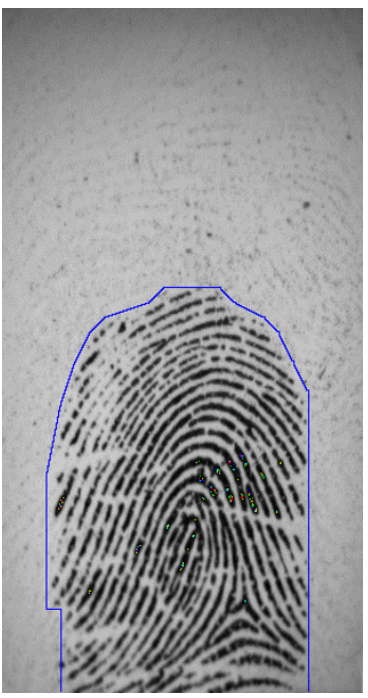

(c)

FIgURE 26: Segmentation results of three fingerprints from FVC2002 DB2: (a) is from the training data, (b) and (c) are from the test data.

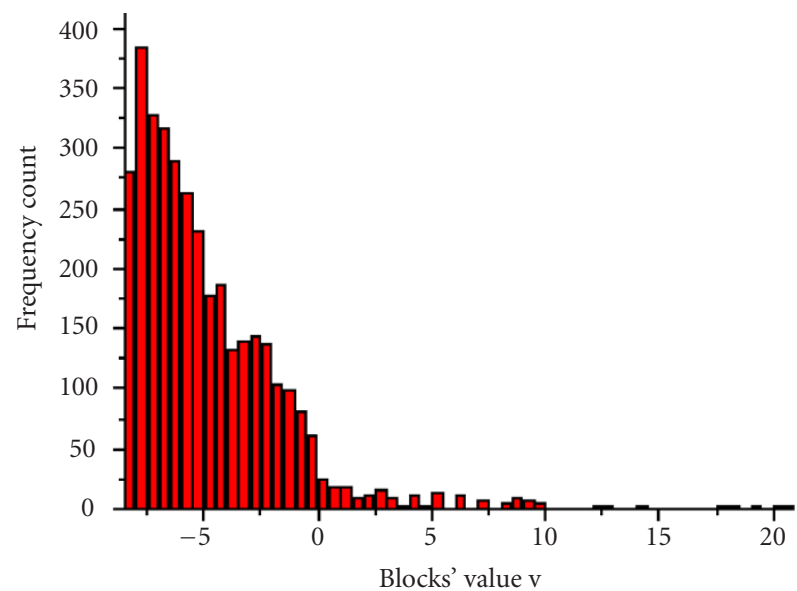

(a)

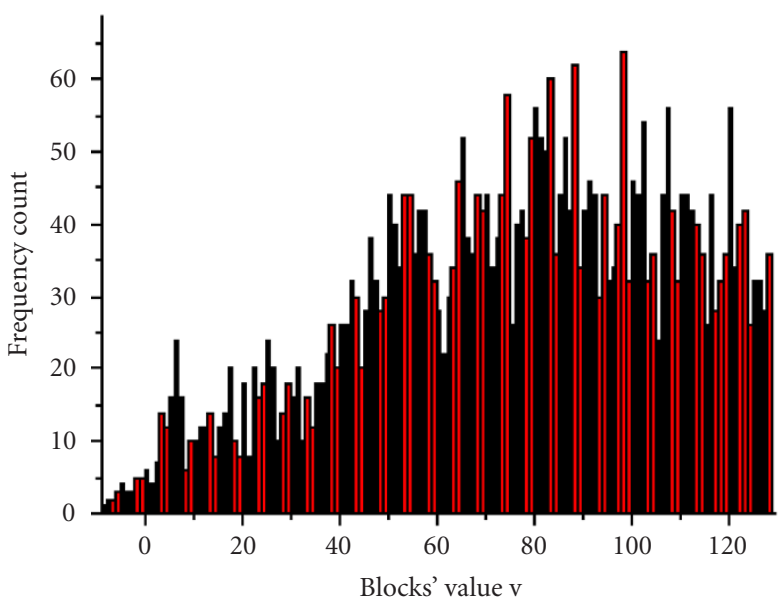

(b)

FIGURE 27: The value of the (a) background and (b) foreground class in linear classification in FVC2002 DB4.

The error rate of DB4 is the following:

$$
\begin{aligned}
p\left(\omega_{0} \mid \omega_{1}\right) & =\frac{24}{4060}=0.0059 \\
p\left(\omega_{1} \mid \omega_{0}\right) & =\frac{95}{3620}=0.0262 \\
\text { Err } & =\frac{119}{7680}=0.0155
\end{aligned}
$$

\subsection{Summary on FVC2002}

From Table 5, it can be seen that the four classifiers assign most importance to CluD. From this point, we can get that the feature of block clusters degree CluD play an important role in classification. From Table 6, we can conclude that our algorithm has excellent classification performance. In the database of FVC2002, only $2.45 \%$ of the blocks are misclassified, while the postprocessing further reduces this ratio. Compared with [1], experimental results show that our algorithm is better than [1]. Human inspection has shown that our algorithm provides accurate high-resolution segmentation results.

\subsection{Segmentation of other fingerprints}

The proposed algorithm is also used to segment the fingerprints of National Institute of Standards and Technology (www.nist.gov). Figure 29 shown two examples of segmented fingerprints of NIST 27. Human inspection shows that the algorithm provides satisfactory results. 
TABLe 5: Results of the linear classifier on FVC2002.

\begin{tabular}{|c|c|c|c|c|}
\hline FVC2002 DB & $\begin{array}{l}\text { Weight vector } \\
\left(w_{0}, w_{1}, w_{2}, w_{3}\right)\end{array}$ & $p\left(\omega_{0} \mid \omega_{1}\right)$ & $p\left(\omega_{1} \mid \omega_{0}\right)$ & $p_{\text {error }}$ \\
\hline DB1 & $(3.723,-0.389,0.071,-12.6)$ & 0.0139 & 0.0225 & 0.0180 \\
\hline DB2 & $(2.342,-0.793,0.046,-11.9)$ & 0.0307 & 0.0268 & 0.0293 \\
\hline DB3 & $(1.152,-0.433,0.067,-24.0)$ & 0.0359 & 0.0347 & 0.0353 \\
\hline DB4 & $(5.701,-0.263,0.036,-10.5)$ & 0.0059 & 0.0262 & 0.0155 \\
\hline Average error rate & & 0.0216 & 0.0275 & 0.0245 \\
\hline
\end{tabular}

TABLE 6: The comparison of error rates of the proposed algorithm and the algorithm in [1] on FVC2002 DB.

\begin{tabular}{lccccc}
\hline \multicolumn{1}{c}{ FVC2002 DB } & DB1 & DB2 & DB3 & DB4 & Average error rate \\
\hline $\begin{array}{l}\text { Classification error rates of } \\
\text { the proposed algorithm }\end{array}$ & 0.0180 & 0.0293 & 0.0353 & 0.0155 & 0.0245 \\
\hline $\begin{array}{l}\text { Classification error rates of } \\
\text { the algorithm in [1] }\end{array}$ & 0.0565 & 0.0659 & 0.0782 & 0.0532 & 0.0635 \\
\hline
\end{tabular}

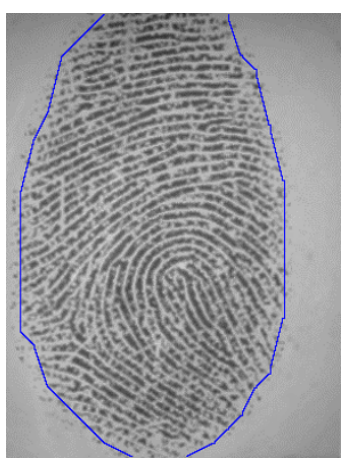

(a)

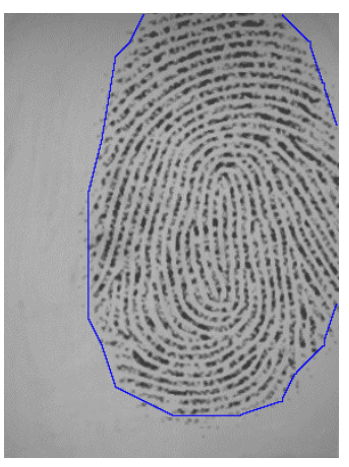

(b)

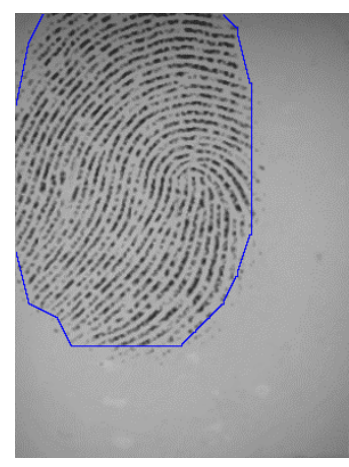

(c)

FIGURE 28: Segmentation results of three fingerprints from FVC2002 DB4: (a) is from the training data, (b) and (c) are from the test data.
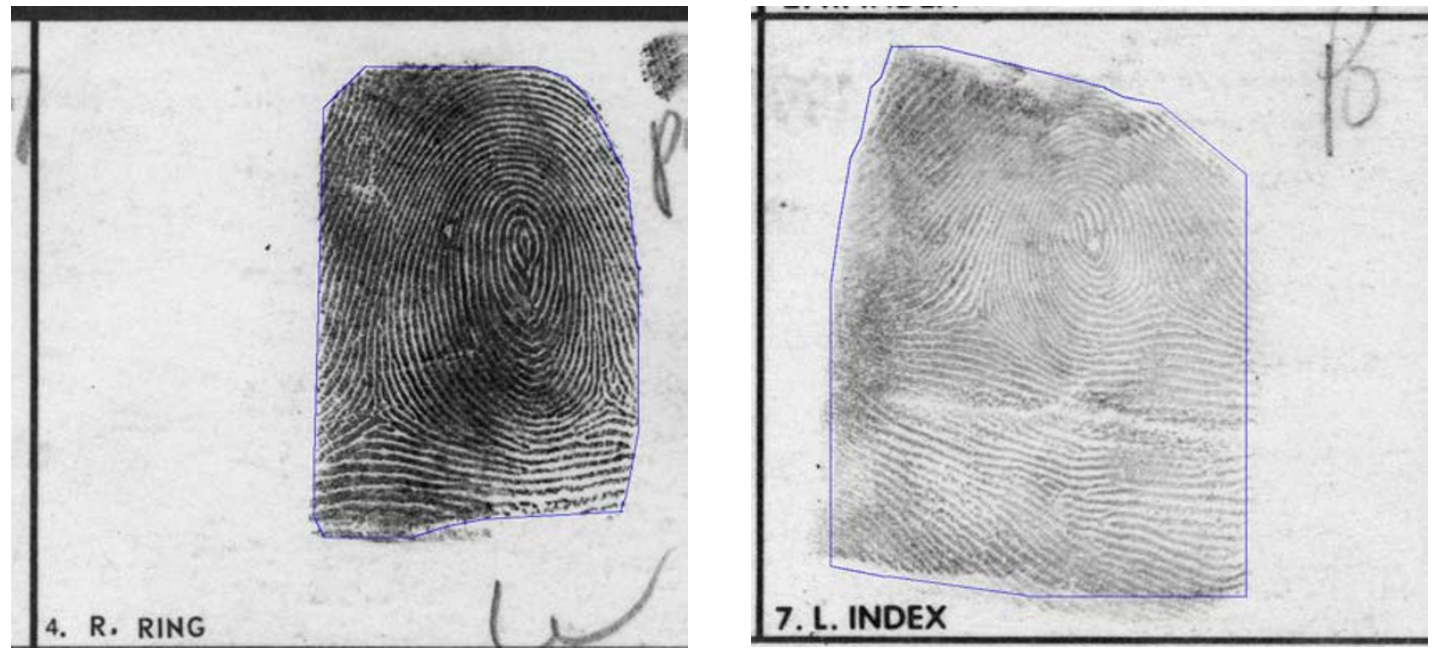

FIGURE 29: Segmentation results of two fingerprints from NIST 27; the size of image is $800 \times 768$. 


\section{CONCLUSIONS AND FUTURE WORKS}

In this paper, an algorithm for the segmentation of fingerprints and a criterion for evaluating the block feature are presented. The segmentation uses three block features, being the block clusters degree, the block mean information, and the block variance. An optimal linear classifier has been trained for the classification per block, the criterion of minimal number of misclassified samples is used. Morphology has been applied as postprocessing to obtain compact clusters and to reduce the number of classification errors.

Human inspection has shown that the proposed method provides accurate high-resolution segmentation results. In the database of FVC2002, only $2.45 \%$ of the blocks are misclassified while the postprocessing further reduces this ratio.

Other texture features of the fingerprint images and the third class representing low quality regions will be investigated in the near future.

\section{ACKNOWLEDGMENT}

This paper is supported by the National Natural Science Foundation of China under Grants 79990580, 69931010, 60172057, 60071002, and 60072007, and the National High Technology Development Program of China under Grant 2001AA144120.

\section{REFERENCES}

[1] A. M. Bazen and S. H. Gerez, "Segmentation of fingerprint images," in ProRISC 2001 Workshop on Circuits, Systems and Signal Processing, Veldhoven, The Netherlands, November 2001.

[2] B. M. Mehtre, N. N. Murthy, S. Kapoor, and B. Chatterjee, "Segmentation of fingerprint images using directional image," Pattern Recognition, vol. 20, no. 4, pp. 429-435, 1987.

[3] B. M. Mehtre and B. Chatterjee, "Segmentation of fingerprint images-A composite method," Pattern Recognition, vol. 22, no. 4, pp. 381-385, 1989.

[4] V. Mosorov, "Using tophat transformation for image fingerprint segmentation," in International Conference on Signals and Electronic Systems, Lodz, Poland, September 2001, pp. 241-246.

[5] D. Maio, D. Maltoni, R. Cappelli, J. L. Wayman, and A. K. Jain, "FVC2002: fingerprint verification competition," Biolab internal Tech. Rep., University of Bologna, Italy, 2002, http://bias.csr.unibo.it/fvc2002/.

[6] A. K. Jain, R. P. W. Duin, and J. Mao, "Statistical pattern recognition: A review," IEEE Trans. on Pattern Analysis and Machine Intelligence, vol. 22, no. 1, pp. 4-37, 2000.

[7] Z. Bian and X. Zhang, Pattern Recognition, Tsinghua University Press, Beijing, China, 2nd edition, 2000.

[8] G. Nagaraja and G. Krishna, "An algorithm for the solution of linear inequalities," IEEE Trans. on Computers, vol. 23, no. 4, pp. 421-427, 1974.

[9] X. P. Luo and J. Tian, "Knowledge based fingerprint image enhancement," in Proc. IEEE International Conference on Pattern Recognition (ICPR '00), Barcelona, Spain, September 2000, vol. 4, pp. 783-786.

[10] A. K. Jain, Fundamentals of Digital Image Processing, PrenticeHall, NJ, USA, 1989.
Xinjian Chen received his B.S. degree in geophysics from Central South University of Technology, China, in 2001. Now he is a candidate for Ph.D. degree at the Institute of Automation, Chinese Academy of Sciences. His research interests include pattern recognition, machine learning, and image processing and their applications in biometrics.

Jie Tian received his Ph.D. degree (with honor) in artificial intelligence from the Institute of Automation, Chinese Academy of Sciences in 1992. From 1994 to 1996, he was a postdoctoral fellow at the medical image processing group, University of Pennsylvania. Sine 1997, he has been a Professor in the Institute of Automation, Chinese Academy of Sciences. His research interests are bioinformatics, medical image process and analysis, pattern recognition, and so forth.

Jiangang Cheng received his B.S. and M.S. degrees in mathematics from Dalian University of Technology, China, in 1998 and 2001 , respectively. Now he is a candidate for Ph.D. degree at the Institute of Automation, Chinese Academy of Sciences. His research interests include pattern recognition, machine learning, and image processing and their applications in biometrics.

Xin Yang received her Ph.D. degree in precision instrument from Tianjig University in 2000. Now she is a postdoctoral fellow in the Institute of Automation, Chinese Academy of Sciences. Her research interests are pattern recognition, medical image process and analysis, and so forth.
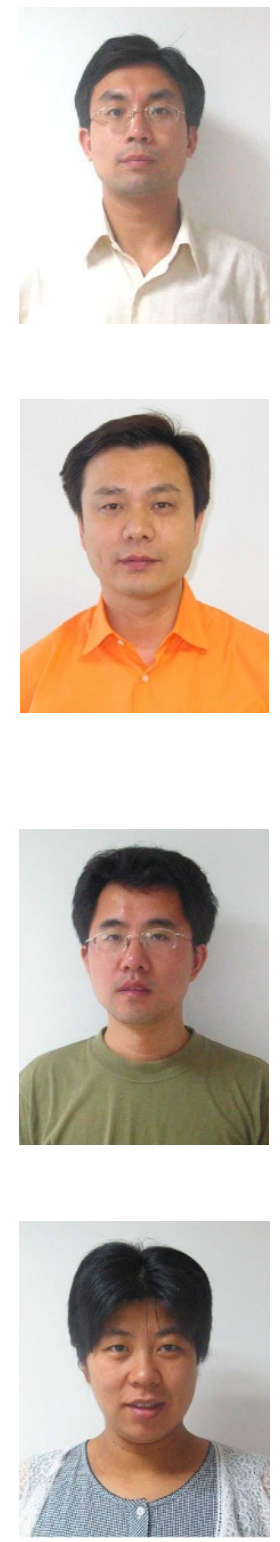